\title{
Renal Lymphoma with Mesenteric Lymphomatosis in a Cat
}

\author{
Jin-ha Jeon*, Doo-won Song*, Woong-bin Ro*, Heyong-seok Kim*, Ga-won Lee*, Jun-ho Cho**, \\ Woo-chang Jeong ${ }^{* * *}$, Soo-hyeon Kim ${ }^{* * * *}$, Jung-hyang Sur**** and Hee-myung Park ${ }^{* 1}$ \\ *Department of Veterinary Internal Medicine, College of Veterinary Medicine, Konkuk University, Seoul 05029, Korea \\ **Goodpapa Animal Hospital, Seoul 07008, Korea \\ ***Africa Animal Medical Center, Seoul 07590, Korea \\ ****Department of Veterinary Pathology, College of Veterinary Medicine, Konkuk University, Seoul 05029, Korea
}

(Received: May 07, 2020 / Revised: June 25, 2020 / Accepted: July 06, 2020)

\begin{abstract}
A 6-year-old castrated male Russian Blue cat was presented for evaluation of dyschezia. Abdominal ultrasound revealed hyperechoic nodules in both kidneys, heterogeneous mass in abdomen, and extensive mesenteric thickening with multiple hypoechoic nodules. Computed tomography showed multiple hypodense lesions in both kidneys and diffuse nodular infiltration around the mesentery. Fine needle aspirates (FNA) acquired under ultrasound guidance from the mesentery consisted of large lymphocytes which have round to irregular nuclei with granular chromatin, prominent nucleoli and a small amount of basophilic cytoplasm. Polymerase chain reaction (PCR) for antigen receptor gene rearrangement result of FNA sample revealed a T-cell malignancy. The cat died from acute renal failure after 1 cycle of modified Madison-Wisconsin L-CHOP protocol. Postmortem examination revealed bilaterally enlarged lumpy-bumpy shaped kidneys. Histopathologic examination showed an infiltration of malignant lymphocytes into the renal parenchyma and mesentery. Immunohistochemical staining of the renal sample displayed a negative expression of CD3, PAX5, MUM-1, and CD79. The clinical features and prognosis of the cat with renal lymphoma with mesenteric lymphomatosis have been described in this report.
\end{abstract}

Key words : feline, lymphoma, acute renal failure, lymphomatosis.

\section{Introduction}

Lymphoma is one of the most common lymphoproliferative disorders, representing 50 to $90 \%$ of all hematopoietic tumors in cats (27). Among lymphomas, renal lymphoma is the second most extranodal lymphoma in cats (26). It can be presented as primary renal lymphoma or occur concurrent with other alimentary lymphomas $(11,25)$. In general, more than half of cats with renal lymphoma have signs of renal insufficiency (13).

In spite of the high prevalence of feline lymphoma, peritoneal dissemination of lymphoma (lymphomatosis) has been rarely reported. The mechanism of invasion is unknown since there is no lymphoid tissue in peritoneum as previously decribed (14). Most renal lymphoma constituted a Bcell immunophenotype (6). In humans, non-B non-T lymphomas are referred to lymphoma lacks both b-cell and t-cell markers, which usually exhibit poor prognosis (9). This report decribes a cat with renal lymphoma with mesenteric lymphomatosis, which showed negative expression to both B-cell and T-cell markers.

\section{Case}

A 6-year-old castrated male Russian Blue cat presented

${ }^{1}$ Corresponding author.

E-mail : parkhee@konkuk.ac.kr with dyschezia. A physical examination revealed low body condition score $(2 / 5)$ and mass was palpated by abdominal examination. There were no remarkable findings in complete blood cell count, peripheral blood smear, and serum biochemical tests. Abdominal radiograph exhibited ill-defined round soft tissue opacity mass cranial to bladder (Fig 1). The following are abdominal ultrasonographic findings of the cat (Fig 2). The mass contained heterogeneous echotexture with mixed echogenicity and irregular discontinuous margin, which

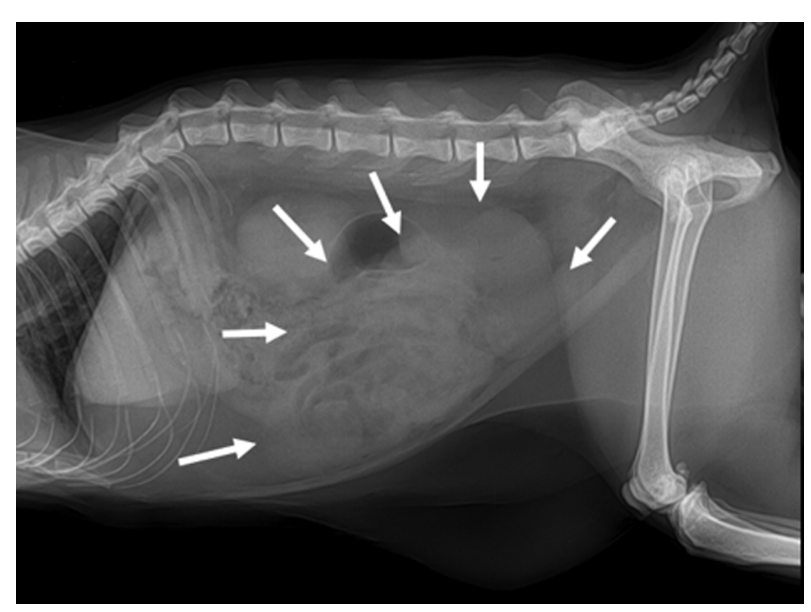

Fig 1. Right lateral radiograph of abdominal cavity in a cat diagnosed as renal lymphoma with mesenteric lymphomatosis. Illdefined round soft tissue opacity mass (white arrows) is shown on middle to caudal abdomen. 


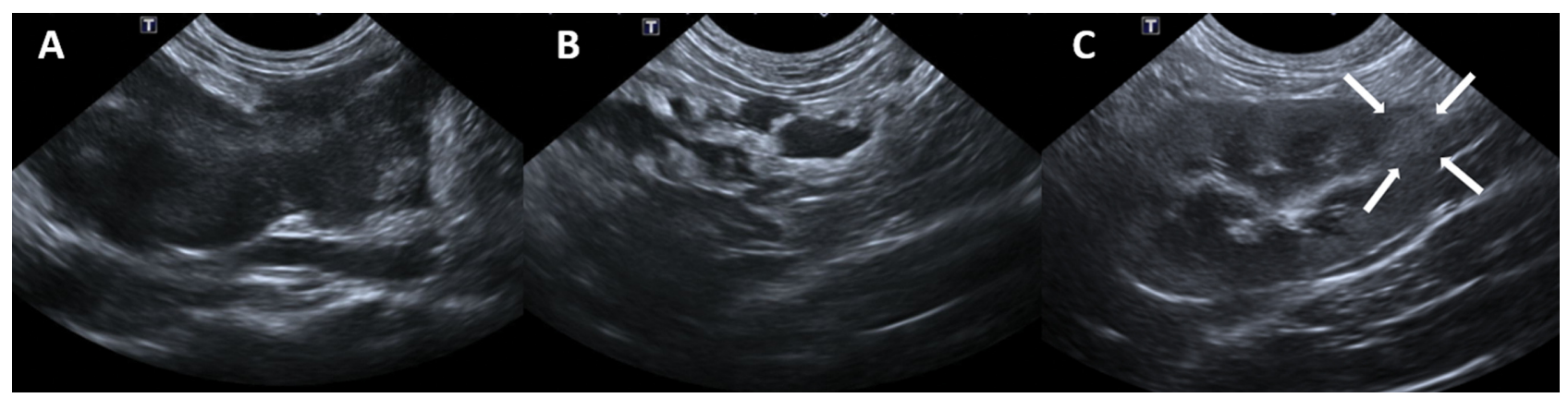

Fig 2. Abdominal ultrasonographic findings of the cat diagnosed as renal lymphoma with mesenteric lymphomatosis. (A) The heterogeneous mass infiltrated into mesentery. (B) Diffuse and extensive mesenteric thickening with multiple hypoechoic nodules/plaques arising from the mesentery. (C) Hyperechoic nodules in both kidneys were definitely diagnosed as lymphoma infiltrated to the kidney based on histology (white arrows).
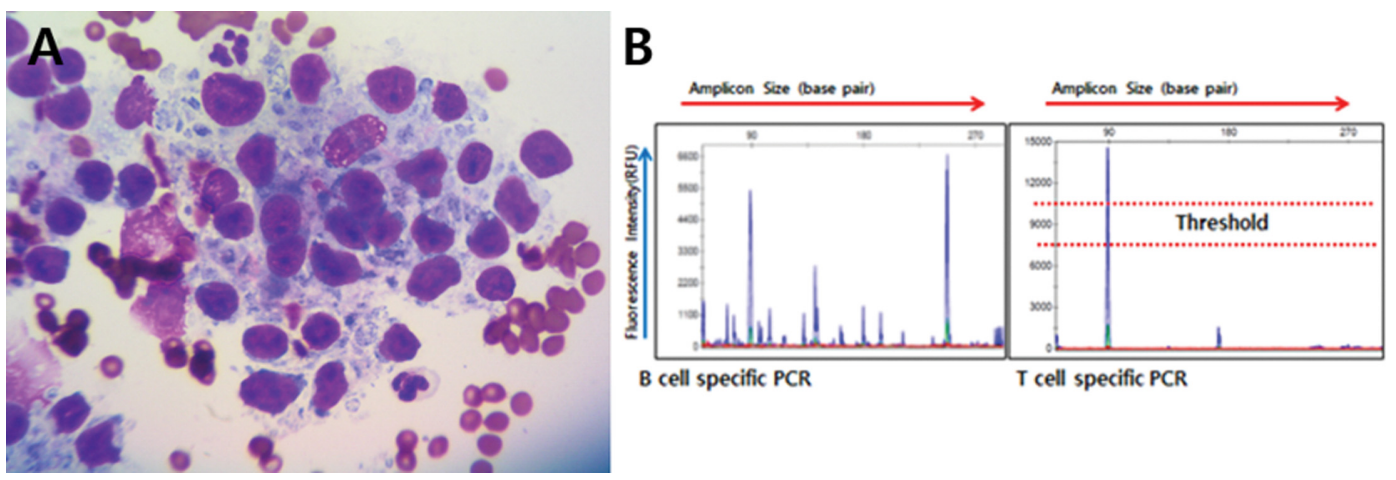

Fig 3. Ultrasound-guided fine needle aspiration biopsy from the cat diagnosed as renal lymphoma with mesenteric lymphomatosis. (A) Large atypical round cells with round to irregular nuclei, basophilic cytoplasm, prominent nucleoli were seen on cytologic examination (Diff-Quik, magnification $=\mathrm{x} 1000$ ). (B) Molecular clonality PCR testing by capillary electrophoresis of FNA sample showed monoclonal peak of $\mathrm{T}$ cells.

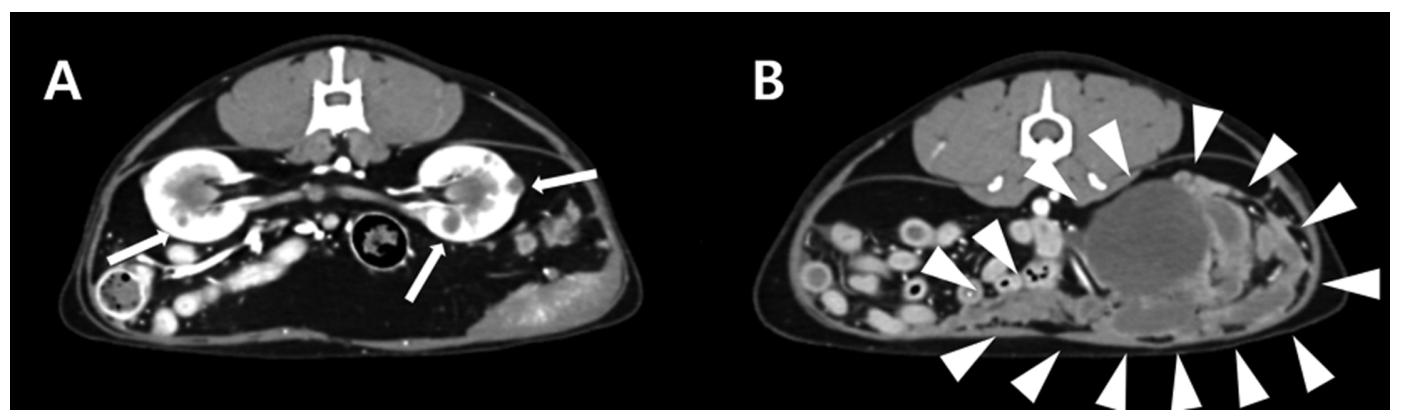

Fig 4. Computed tomographic images in a cat diagnosed as renal lymphoma with mesenteric lymphomatosis. (A) Multiple hypodense lesions were found in both kidneys (white arrows). (B) Note that abdominal mass and diffuse nodular infiltration around the mesentery (white arrowheads).

infiltrated to mesentery. The mesenteric serosal border of this case was irregular and poorly ill defined, and coalesced with thickened hypoechoic and/or anechoic mesenteric tissues. Additionally, multiple discrete-to-coalescing hypoechoic lesions were observed in the visceral and parietal peritoneum. Hyperechoic nodules in both kidneys were also detected. Ultrasound guided fine-needle aspirate of the mesenteric lesion showed large atypical round cells with basophilic cytoplasm, irregular nuclear membrane (Fig 3). According to cytologic evaluation, it was considered that the abdominal mass was originated from malignant lymphoid tissues. Thus, polymerase chain reaction for antigen receptor rearrange- ment (PARR) was conducted to determine cell lineage and assess clonality to distinguish neoplastic from inflammatory lymphoid cells. Computed tomography (CT) was performed to evaluate local or distant metastasis, and set therapeutic plan appropriate to this cat.

CT revealed the abdominal mass, and diffuse nodular infiltration around the mesentery. Multiple hypodense lesions were found in both kidneys (Fig 4). Molecular clonality PCR testing by capillary electrophoresis (3730xl DNA Analyzer, GeneScan $^{\text {TM }} 500$ LIZ $^{\mathrm{TM}}$ dye Size Standard, GeneMapper ${ }^{\mathrm{TM}}$ Software 5, Thermo Fisher Scientific, USA) result of mesenteric FNA sample showed monoclonal expansion of $\mathrm{T}$ cells 
(Fig 3). Thus, high grade T-cell lymphoma was tentatively diagnosed.

Because of biologic aggressiveness, surgical removal of the mass was not considered. Instead, oral chlorambucil ( $2 \mathrm{mg} / \mathrm{cat}$ PO EOD) and prednisolone (initially $2 \mathrm{mg} / \mathrm{kg}$ PO daily, reduced to $1 \mathrm{mg} / \mathrm{kg}$ EOD) chemotherapy was initiated. During 1 month after chemotherapy, ultrasonographic findings showed gradual decrease in mass size. However, lymphoma was relapsed on day 54. Thus, Modified MadisonWisconsin L-CHOP protocol was used as rescue chemotherapy. After 1 cycle of L-CHOP protocol, abdominal mass completely disappeared in abdominal ultrasound. However, irregular lumpy-bumpy shape and hypoechoic subcapsular thickening was detected in bilateral kidney. Hematological examination showed anemia (HCT 22.6\%; reference, 25.0$50.0 \%$ ), and serum biochemical profiles revealed increase in serum creatinine concentration $(4.05 \mathrm{mg} / \mathrm{dL}$; reference, 0.8 $1.8 \mathrm{mg} / \mathrm{dL})$. Despite aggressive fluid therapy, this cat died in day 91 .

Postmortem examination revealed bilaterally enlarged kidneys. There were multifocal, nodular lymphomatous infiltrates throughout the cortex, which was swollen (Fig 5). Histopathological examination revealed malignant lymphocytic infiltration in both kidneys and mesentery (Fig 6). Thus, renal lymphoma was diagnosed, which have metastasized to the mesentery. Immunohistochemical staining (IHC) of the biopsy sample of the kidney showed a negative expression with markers of both B cell marker: 1) PAX5 (Clone 24/Pax5; BD Biosicences, San Jose, CA, USA); 2) MUM1 (Clone
MUM1p; Dako, Tokyo, Japan); 3) CD79 (Clone HM57; Dako) and T cell marker: CD3 (Dako).

Among extranodal lymphoma, lymphoma in kidney is the second most common form (26). However, there are little information on the immunohistologic classification of renal lymphoma; In a previous study (6), majority constituted a Bcell immunophenotype. And, in Australia (6), most renal lymphoma was B-cell type and intermediate to high grade. However, in this case, PARR result of mesenteric FNA sample revealed T-cell malignancy, and IHC result of kidney sample showed a negative expression with markers of both $\mathrm{B}$ cell and T cell. Previous studies $(21,22)$ described feline gastrointestinal large granulocyte lymphocyte (LGL) lymphoma cases which were nonreactive for both $\mathrm{B}$ - and T-cell markers. Feline non-B non-T LGL lymphoma is usually assumed to be a NK cell lymphoma because NK cells lack are CD3 and T-cell receptor. However, there is no specific marker for feline NK cells, thus confirmation of cell origin is not feasible. PAX5 expression is used to differentiate the classic form from the atypical form in hodgkin lymphoma of humans, and people who lacks PAX5 expression has a less favorable prognosis. $(5,8)$. Although it can be assumed that lack of PAX5 expression acts as a poor prognostic factor in feline lymphoma also (24), further studies are necessary to support this assumption. And, although IHC can be a valuable tool, a negative stain does not always exclude a specific cell type because technical difficulties or tumor cell dedifferentiation can cause loss of expression of expected proteins or markers, and it may result in a negative stain (4). Moreover, PARR was

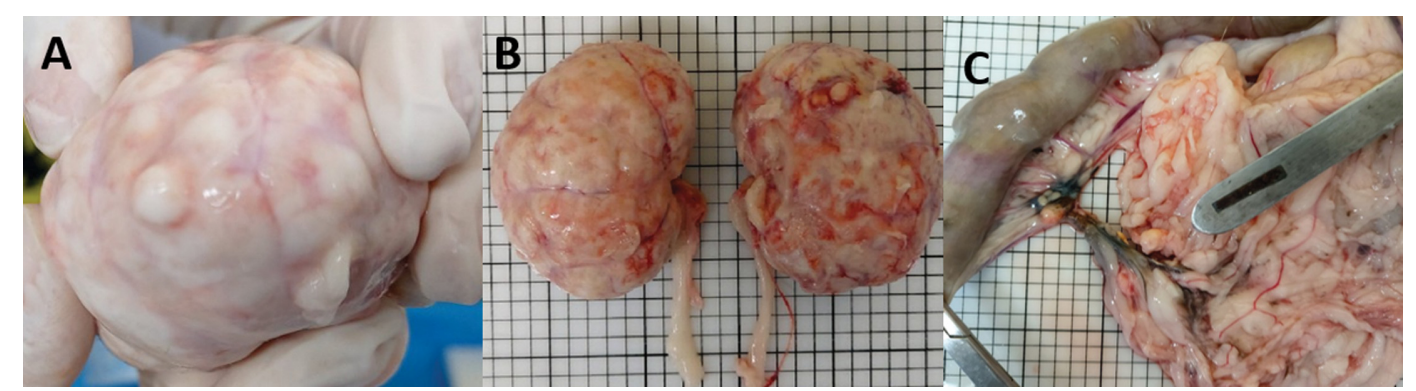

Fig 5. Postmortem findings of the cat diagnosed as renal lymphoma with mesenteric lymphomatosis. (A) Grossly, kidney was lumpybumpy due to multiple nodules. (B) When decapsulated, bilateral kidney showed severe congestion. (C) Multiple nodules were detected throughout the mesentery.

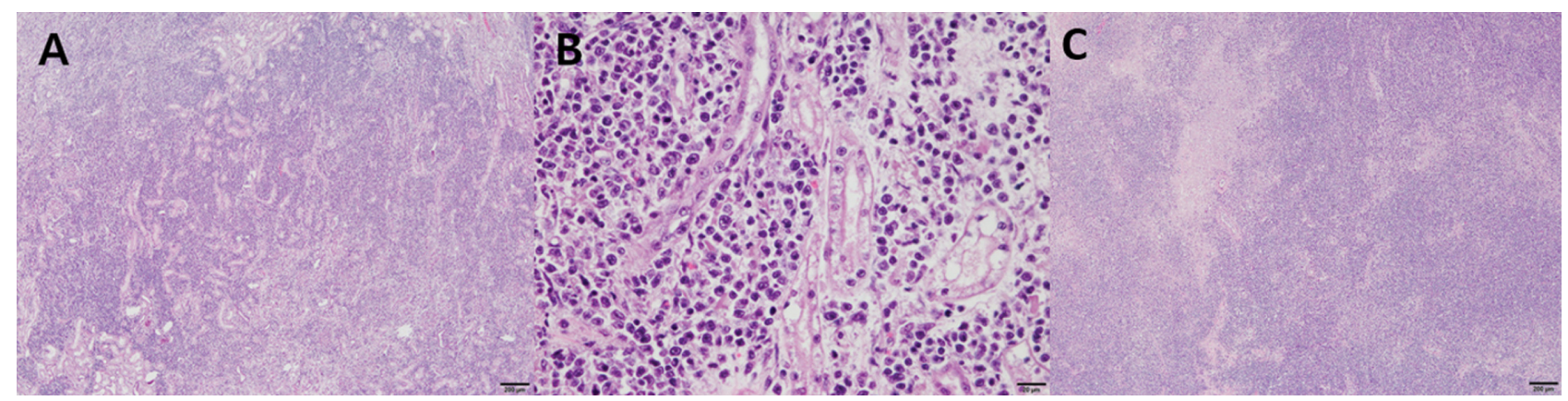

Fig 6. Histopathologic findings of the cat diagnosed as renal lymphoma with mesenteric lymphomatosis. (A) Malignant lymphocytes invaded around the left renal tubule, glomerulus, and collecting duct. (B) Neoplastic lymphocytes in kidney showed remarkable anisokaryosis and anisocytosis. (C) Diffuse invasion of malignant lymphocytes throughout the mesentery (H\&E, original magnification 40x. (A, C) and 400x (B). Bar $=200 \mu \mathrm{m}(\mathrm{A}, \mathrm{C})$ and $20 \mu \mathrm{m}(\mathrm{B}))$. 
performed by mesenteric FNA sample, not a renal FNA sample. As the cancer progress, tumor cells can adopt expression of different lineage markers, which mean multiple level of heterogeneity of cancer (16). Within the same tumors, tumor cells may exist in different states of differentiation, which can be identified by different cell surface markers (29). Taken together the following may be the reasons for discrepancy between result of PARR and IHC: 1) Technical problem; 2) differences in analysis site (mesentery and kidney); 3) Losses or changes of surface markers due to tumor cell dedifferentiation.

In humans, the spread of lymphoma to the peritoneum, omentum, and mesenteries is termed peritoneal lymphomatosis (18). In contrast, there are only a few reports of lymphomatosis in veterinary medicine $(1,7,11,19,25,30)$. Hypoechoic lesions infiltrating the mesentery, and/or mesenteric or visceral peritoneal masses were reported as ultrasonographic findings on this phenomenon $(1,12,19,20)$. These ultrasonographic features were also identified in this case. In human medicine, CT is mainly used to characterize neoplastic, infectious, or inflammatory peritoneal pathology $(2,10)$. Some CT findings of peritoneal lymphomatosis in human medicine are recognized: the replacement of normal mesenteric fat to softtissue tumor, discrete or confluent nodules, mesenteric mass, and characteristic pleated and stellate patterns caused by abnormal fixation of small bowels due to stiff and retractile mesentery (3). In veterinary medicine, ultrasonography is more commonly used than CT in the investigation and differentiation of abdominal lesions due to the higher cost of the CT (17). To the authors' knowledge, the CT appearance of mesenteric lymphomatosis in veterinary medicine has not been reported. In this case, mesenteric mass and the combination of small nodules and finely infiltrated fat was detected in CT.

Acute renal failure of in this cat may due to progressive renal lymphoma, or nephrotoxicity of doxorubicin. Nephrotoxicity is commonly encountered in cats, and renal function should be monitored closely prior to and during doxorubicin therapy (27). However, previous studies using combination protocols reported the addition of doxorubicin resulted in more durable responses $(13,28)$. Moreover, CHOP-based protocols are generally appropriate for cats with large cell type, intermediate- to high-grade lymphoma involving any anatomic site, including renal lymphoma (27).

\section{Conclusions}

This report describes the cat with an abdominal mass diagnosed as primary renal lymphoma and mesenteric metastasis. In this case, the chief complaint was dyschezia, which was caused by abdominal mass. However, the cat died from acute renal failure in spite of removal of abdominal mass. Although peritoneal lymphomatosis is rare in feline lymphoma, it should be included as a differential diagnosis in cats with other nodal or extranodal lesions. In addition, precise diagnosis including histopathologic evaluation of kidney is needed to early detection of acute renal failure when there are lesions in the kidney. Finally, negative expression of both B-cell and T-cell surface markers may be related to poor prognosis in cats.

\section{Acknowledgements}

This research was supported by the Bio \& Medical Technology Development Program of the National Research Foundation (NRF) funded by the Korean government (MSIT) (2016M3A9B6903437). The authors thank to Dr. Woo-chang Jeong for excellent imaging support and Dr. Soo-hyeon Kim for histopathology services. The authors thank to Pobanilab Laboratories (Guri-si, Gyenggi-do, Korea) for molecular diagnostic services.

\section{References}

1. Benigni L, Lamb CR, Corzo-Menendez N, Holloway A, Eastwood JM. Lymphoma affecting the urinary bladder in three dogs and a cat. Vet Radiol Ultrasound 2006; 47: 592596.

2. Cooper C, Jeffrey RB, Silverman PM, Federle MP, Chun GH. Computed tomography of omental pathology. J Comput Assist Tomogr 1986; 10: 62-66.

3. Diop AD, Fontarensky M, Montoriol PF, Da Ines D. CT Imaging of peritoneal carcinomatosis and its mimic. Diagnostic and Interventional Imaging 2014; 95: 861-872.

4. Ehrhart EJ, Debra A. Kamstock, Barbara E. Powers. The pathology of neoplasia. In: Withrow and MacEwen's Small Animal Clinical Oncology. 5th ed. WB Saunders. 2013: 5167.

5. Feldman AL, Dogan A. Diagnostic uses of Pax5 immunohistochemistry. Adv Anat Pathol 2007; 14: 323-334.

6. Gabor LJ, Canfield PJ, Malik R. Immunophenotypic and histological characterization of 109 cases of feline lymphosarcoma. Aust Vet J 1999; 77: 436-441.

7. Gabor LJ, Malik R, Canfield PJ. Clinical and anatomical features of lymphosarcoma in 118 cats. Aust Vet J 1998; 76: 725-732.

8. Jensen KC, Higgins JP, Montgomery K, Kaygusuz G, van de Rijn M, Natkunam Y. The utility of PAX5 immunohistochemistry in the diagnosis of undifferentiated malignant neoplasms. Mod Pathol 2007; 20: 1-7.

9. Karube K, Ohshima K, Tsuchiya T, Yamaguchi T, Suefuji H, Suzumiya J, Harada M, Kikuchi M. Non-B, non-T neoplasms with lymphoblast morphology: further clarification and classification. Am J Surg Pathol 2003; 27: 1366-1374.

10. Kim Y-S, Cho O-K, Song S-Y, Lee H-S, Rhim H-C, Koh B-H. Peritoneal lymphomatosis: CT findings. Abdom Imaging 1998; 23: 87-90.

11. Little L, Patel R, Goldschmidt M. Nasal and nasopharyngeal lymphoma in cats: 50 cases (1989-2005). Vet Pathol 2007; 44: 885-892.

12. Monteiro CB, O'Brien RT. A retrospective study on the sonographic findings of abdominal carcinomatosis in 14 cats. Vet Radiol Ultrasound 2004; 45: 559-564.

13. Mooney SC, Hayes AA, Matus RE, MacEwen EG. Renal lymphoma in cats: 28 cases (1977-1984). J Am Vet Med Assoc 1987; 191: 1473-1477.

14. Moore AS, Cotter SM, Frimberger AE, Wood CA, Rand WM, L'Heureux DA. A comparison of doxorubicin and COP for maintenance of remission in cats with lymphoma. J Vet Intern Med 1996; 10: 372-375.

15. Morgan KRS, North CE, Thompson DJ. Sonographic features of peritoneal lymphomatosis in 4 cats. J Vet Intern Med 2018; 32: 1178-1184.

16. Morvinski DF, Verma IM. Dedifferentiation and reprogramming: 
origins of cancer stem cells. EMBO rep 2014; 15: 244-253.

17. Oetelaar GS, Lim CK, Heng HG, Fulkerson CM, Shaevitz $\mathrm{MH}$, Thompson CA. Ultrasonographic features of colonic Bcell lymphoma with mesenteric lymphomatosis in a cat. Vet Radiol Ultrasound 2018 Jul 16; doi: 10.1111/vru.12665.

18. O'Neill AC, Shinagare AB, Rosenthal MH, Tirumani SH, Jagannathan JP, Ramaiya NH. Differences in CT features of peritoneal carcinomatosis, sarcomatosis, and lymphomatosis: Retrospective analysis of 122 cases at a tertiary cancer institution. Clin Radiol 2014; 69: 1219-1227.

19. Penninck DG, d'Anjou MA. Gastrointestinal tract. Atlas of Small Animal Ultrasonography. 2nd ed. Ames: Wiley Blackwell. 2015: 290

20. Penninck DG, Moore AS, Tidwell AS, Matz ME, Freden GO. Ultrasonography of alimentary lymphosarcoma in the cat. Vet Radiol Ultrasound 1994; 35: 299-306.

21. Pohlman LM, Higginbotham ML, Welles EG, Johnson CM. Immunophenotypic and histologic classification of 50 cases of feline gastrointestinal lymphoma. Vet Pathol 2009; 46: 259-268.

22. Roccabianca P, Vernau W, Caniatti M, Moore PF. Feline large granular lymphocyte (LGL) lymphoma with secondary leukemia: primary intestinal origin with predominance of a CD3/ CD8(alpha)(alpha) phenotype. Vet Pathol. 2006; 43: 15-28.

23. Sheth S, Horton KM, Garland MR, Fishman EK. Mesenteric neoplasms: CT appearances of primary and secondary tumors and differential diagnosis. Radiographics 2003; 23: 457-473.

24. Sierra M, Viera R, Ferreira M, Pascoli A, Reis N, Jark P, Hernandez GV, Costa MT, Nardi AD, Calazans S. Cutaneous lymphoma of the tarsus in a cat-case study. Revista MVZ Córdoba 2017; 22: 5747-5754.

25. Takahashi R, Goto N, Ishii H, Ogiso Y, Saegusa J. Pathological observations of natural cases of feline lymphosarcomatosis. Nippon Juigaku Zasshi 1974; 36: 163-173.

26. Taylor SS, Goodfellow MR, Browne WJ, Walding B, Murphy S, Tzannes S, Gerou-Ferriani M, Schwartz A, Dobson JM. Feline extranodal lymphoma: response to chemotherapy and survival in 110 cats. J Small Anim Pract 2009; 50: 584-592.

27. Vail DM. Feline lymphoma and leukemia. In: Withrow SJ, Vail DM, Page RL, eds. Small Animal Clinical Oncology. Missouri, USA: Elsevier Saunders. 2013: 638-653.

28. Vail DM, Moore AS, Ogilvie GK, Volk LM. Feline lymphoma (145 cases): Proliferation indices, CD3 immunoreactivity and their association with prognosis in 90 cats receiving therapy. $\mathrm{J}$ Vet Intern Med 1998; 12: 349-354.

29. Visvader JE, Lindeman GJ. Cancer stem cells: current status and evolving complexities. Cell Stem Cell 2012; 10: 717-728.

30. Whitman JJ, Cockrell KO, Hall WT, Gilmore CE. An unusual case of feline leukemia and an associated syncytium-forming virus. Am J Vet Res 1975; 36: 873-880. 